\title{
Effect of community-based distribution of misoprostol on facility delivery: a scoping review
}

\author{
Gizachew Tadele Tiruneh ${ }^{1,2^{*}}$ (D) Bereket Yakob ${ }^{2,3}$, Wubegzier Mekonnen Ayele ${ }^{2,4}$, Muluneh Yigzaw ${ }^{2,5}$, \\ Meselech Assegid Roro ${ }^{2,4}$, Araya Abrha Medhanyi ${ }^{2,6}$, Etenesh Gebreyohannes Hailu ${ }^{2,7}$ and \\ Yibeltal Tebekaw Bayou ${ }^{1,2}$
}

\begin{abstract}
Introduction: Community distribution of misoprostol to pregnant women in advance of labor is one of the compelling strategies for preventing postpartum hemorrhage. Concerns have been reported that misoprostol distribution could reduce facility delivery or lead to misuse of the medication. This scoping review was conducted to synthesize the evidence on the effect of community-based misoprostol distribution on rates of facility delivery, and to assess the frequency of mothers taking distributed misoprostol before delivery, and any harmful outcomes of such misuse.
\end{abstract}

Methods: We included peer-reviewed articles on misoprostol implementation from PubMed, Cochrane Review Library, Popline, and Google Scholars. Narrative synthesis was used to analyze and interpret the findings, in which quantitative and qualitative syntheses are integrated.

Results: Three qualitative studies, seven observational studies, and four experimental or quasi-experimental studies were included in this study. All before-after household surveys reported increased delivery coverage after the intervention: ranging from 4 to 46 percentage points at the end of the intervention when compared to the baseline. The pooled analysis of experimental and quasi-experimental studies involving 7564 women from four studies revealed that there was no significant difference in rates of facility delivery among the misoprostol and control groups [OR 1.011; 95\% Cl: 0.906-1.129]. A qualitative study among health professionals also indicated that community distribution of misoprostol for the prevention of postpartum hemorrhage is acceptable to community members and stakeholders and it is a feasible interim solution until access to facility birth increases.

In the community-based distribution of misoprostol programs, self-administration of misoprostol by pregnant women before delivery was reported in less than $2 \%$ of women, among seven studies involving 11,108 mothers. Evidence also shows that most women who used misoprostol pills, used them as instructed. No adverse outcomes from misuse in either of the studies reviewed.

Conclusions: The claim that community-based distribution of misoprostol would divert women who would have otherwise had institutional deliveries to have home deliveries and promote misuse of the medication are not supported with evidence. Therefore, community-based distribution of misoprostol can be an appropriate strategy for reducing maternal deaths which occur due to postpartum hemorrhages, especially in resource-limited settings.

Keywords: Community-based distribution, Diversion of facility birth, Facility delivery, Misoprostol

\footnotetext{
* Correspondence: gizt121@gmail.com

'JSI Research \& Training Institute, Inc./ The Last Ten Kilometers (L10K) Project, Addis Ababa, Ethiopia

${ }^{2}$ Members of the National Reproductive, Maternal, Newborn, Child,

Adolescent Health, and Nutrition (RMNCAH-N) Research Advisory Council

(RAC), Addis Ababa, Ethiopia

Full list of author information is available at the end of the article
}

(c) The Author(s). 2019 Open Access This article is distributed under the terms of the Creative Commons Attribution 4.0 International License (http://creativecommons.org/licenses/by/4.0/), which permits unrestricted use, distribution, and reproduction in any medium, provided you give appropriate credit to the original author(s) and the source, provide a link to the Creative Commons license, and indicate if changes were made. The Creative Commons Public Domain Dedication waiver (http://creativecommons.org/publicdomain/zero/1.0/) applies to the data made available in this article, unless otherwise stated. 


\section{Introduction}

Maternal mortality ratios (MMR) remain high in lowand-middle-income countries (LMICs), and reduction of MMR continues to be a priority challenge in the Sustainable Development Goals (SDG) era [1]. Accordingly, to achieve the SDG of reducing the global MMR to 70 per 100,000 live births by 2030, LMICs needs to implement innovative and high impact interventions aimed at preventing and managing the main causes of maternal deaths and providing high-quality services in the continuum of maternity care $[2,3]$.

A wealth of evidence shows that hemorrhage is one of the major causes of maternal mortality [4-7]. More than two-thirds of maternal deaths due to hemorrhage occur during the postpartum period, which accounts for $20 \%$ of all maternal deaths in developing regions [5]. However, in Ethiopia, a systematic review of national evidence shows that $\mathrm{PPH}$ accounts for $30 \%$ of maternal deaths $[8,9]$.

Most maternal deaths occurring due to $\mathrm{PPH}$ are in poorly resourced facilities or outside of a health facility where there is no access to skilled obstetric care [10-12]. Women who deliver at home face the highest risk of $\mathrm{PPH}$, as they do not benefit from the support of skilled birth attendants and are less likely to receive timely care and medications that prevent and manage PPH [12]. Evidence shows that most $\mathrm{PPH}$-associated deaths could be avoided if active management of third stage of labor (AMTSL) is implemented [13], adverse outcomes and complications are prevented or managed using safe drugs in communities and facilities, and effective referral mechanisms are implemented during delivery and in the postpartum period [14]. Intravenous or intramuscular administration of uterotonics are the most essential component of AMTSL [15] and oxytocin remains the first choice uterotonics for the prevention of PPH [14].

Misoprostol distribution at community level to women during pregnancy is one of the interventions for preventing $\mathrm{PPH}$ to reach women who deliver at home without skilled attendant [16-18]. Misoprostol is a generic, inexpensive, heat-stable, and potent uterotonic that can be administered orally, sublingually, rectally, and vaginally [19] for the prevention of PPH. It has considerable advantages over other uterotonics in resource-poor settings to reach woman without access to institutional delivery. Misoprostol has been studied in different setups and is endorsed by the World Health Organization (WHO) as a solution for women who give birth in facilities without oxytocin or where there is low coverage of skilled attendance [16]. Clinical trials have verified the effectiveness and safety of community distribution of misoprostol [14, 17, 20, 21] where access to skilled birth attendance and oxytocin is limited. A pooled estimate of randomized controlled trials (RCT) comparing $600 \mu \mathrm{g}$ of oral or sublingual misoprostol with placebo in primary care or home delivery settings show that misoprostol resulted in 24 and $41 \%$ reductions in the incidence of $\mathrm{PPH}$ and severe $\mathrm{PPH}$ compared with placebo, respectively [17].

Despite the existing evidence, community-based distribution of misoprostol is still the least prioritized intervention in the maternal survival strategies [18, 22-25]. This is due to concerns of policymakers' and practitioners' $[12,17,21,25]$ that misoprostol distribution at community level might decrease facility deliveries, possibly lead to misuse of misoprostol (including taking the drug before delivery, and using the drug for the purpose of inducing abortion), and lack of technologies and expertise to diagnose multiple pregnancies before using it at community levels in resource-limited settings [20, 26]. A range of other barriers at the health system, community, and policy levels are also impeding access to misoprostol for prevention of PPH. These barriers include: 1) absence of registration of misoprostol for the management of PPH $[25,27], 2)$ fear and apprehensions of providers and policymakers regarding its use [25, 27], 3) lack of evidence-based guidelines and provider training [25], 4) inadequate staffing and lack of knowledge and skill of providers regarding causes of $\mathrm{PPH}$, and 5) limited knowledge of the community regarding the appropriate dosage and timing of administration for $\mathrm{PPH}$ presentation and management $[20,27]$.

This scoping review was, therefore, conducted to synthesize the evidence on the effect of communitybased misoprostol distribution in advance of delivery on rates of facility delivery, and to assess the frequency of mothers taking distributed misoprostol before delivery, and any harmful outcomes of such misuse.

\section{Methods \\ Criteria for inclusion}

In this study, researchers used a scoping review methodology to get a wide range of information from both qualitative and quantitative studies. All types of literature on community-distribution of misoprostol for the prevention of $\mathrm{PPH}$ reported in English language were included, with no specification on timing of publication.

\section{Search strategy}

We identified peer-reviewed articles on implementation of community distribution of misoprostol from PubMed, Cochrane Review Library, Popline, and Google Scholars which were made available from February 1-15, 2019. We also applied a snowball approach of searching from the references of papers of the initial search.

The following search strategy was used to search literature from PubMed and CENTRAL databases; 


\section{"(()((C)((()Africa OR Asia OR Caribbean OR West Indies OR South America OR Latin America OR Central America OR Middle East))) OR ((developing countr" OR less developed country * OR under developed country "OR underdeveloped country * OR middle income country * OR low income countr*)))))) AND ((( (postpartum hemorrhage) OR post partum hemorrhage) OR postpartum haemorrhage) OR post partum haemorrhage)) AND misoprostol)) AND ((((community distribution) OR community)) OR community based $)))$ AND (((((adverse effects) OR adverse outcomes)) OR ((misuse) OR ("Drug Misuse"[Mesh] OR "Prescription Drug Misuse"[Mesh]))) OR ((((skilled delivery) OR institutional delivery) OR "Delivery, Obstetric"[Mesh]) OR delivery))".}

Moreover, a combination of terms, including 'misoprostol'; 'misuse'; 'adverse outcomes'; fear of diversion of facility birth'; 'misconceptions'; 'misperceptions'; 'postpartum hemorrhage' (and variations i.e. 'post-partum hemorrhage', 'postpartum hemorrhage'); 'communitybased maternal'; 'maternal health interventions'; 'maternal mortality'; and 'low-income setting', 'developing country', 'resource-poor setting' have been used to identify the required literature from Popline and Google Scholar.

First, any research output with the above-mentioned terms in either the title or abstract of the article was downloaded, and then a combination of these terms was also used to download more resources.

\section{Critical appraisal}

The methodological quality of each study was assessed using the Joanna Briggs Institute (JBI) critical appraisal checklists for different study designs as appropriate [28-30] - to assess the methodological quality of studies and to determine the extent to which included studies have addressed the possibility of bias in its design, conduct, and analysis. Two review authors (GT and MY) independently did appraising the quality of each study included and discrepancies between scores were resolved through discussions.

The quality of the studies was assessed based on the core items recommended for the assessment of methodological quality. To obtain an overall quality score, publications scored " 1 " point for each item fully met and " 0 " for none or very little information reported. Items were given equal weights and a percentage score was generated. Studies that scored $75 \%$ or more were categorized as high quality, scores in the range of $50-74 \%$ were ranked as medium, and scores less than $50 \%$ were rated as poor.

Moreover, standard review protocol, Preferred Reporting Items for Systematic and Meta-Analysis for Scoping
Reviews (PRISMA-ScR) checklist, was followed to establish minimum information that should be included when reviewing and reporting [31]. The protocol, however, was not registered in any databases.

\section{Data extraction and analysis}

The form for abstracting data from reviewed literature was designed and review team members agreed on the contents of the form. Two reviewers (GT and YT) read each identified literature and populated the sheet designed for the purpose. The Preferred Reporting Items for Systematic Reviews and Meta-Analyses (PRISMA) diagram (Fig. 1) was used for the selection of articles to be used in this scoping review.

Facility delivery rate, misuse, adverse effects from misuse of the drug, and misconceptions on the use of misoprostol and fear of diversion of facility delivery to home delivery because of misoprostol's access to mothers were the main points considered in this scoping systematic review.

A narrative synthesis was used to analyze and interpret the findings in which quantitative and qualitative syntheses are integrated. Descriptive information about the eligible studies was summarized using text and tables. Findings from the quantitative resources were narrated thematically followed by findings of qualitative resources. For intervention studies, a random-effects meta-analysis model $[32,33]$ was used to pool the estimates of prevalence of facility birth, accounting for the variability among studies using Stata v15 [34]. The results were presented as average treatment effects (odds ratio) with $95 \%$ confidence intervals.

\section{Results}

\section{Description of studies}

Table 1 presents the characteristics of the studies included in this review. Fourteen studies were included in the review. Seven of the studies were from Africa and the remaining seven were from Asia. Three qualitative studies [12, 26, 42], seven observational studies [35-37, $40,43-45]$, and four experimental or quasi-experimental studies $[38,39,41,46]$ were included in this review. All studies were published from 2006 to 2018.

Interventional activities in observational and experimental studies included training to health workers, antepartum and/or postpartum home visits, identification of pregnant women, provision of prenatal education, community sensitization, and distribution of $600 \mu \mathrm{g}$ misoprostol to women.

\section{Methodological quality of included studies}

According to the JBI quality appraisal tool, two of the RCTs scored high quality (88\%) and a quasiexperimental study scored medium (61\%). On the other 


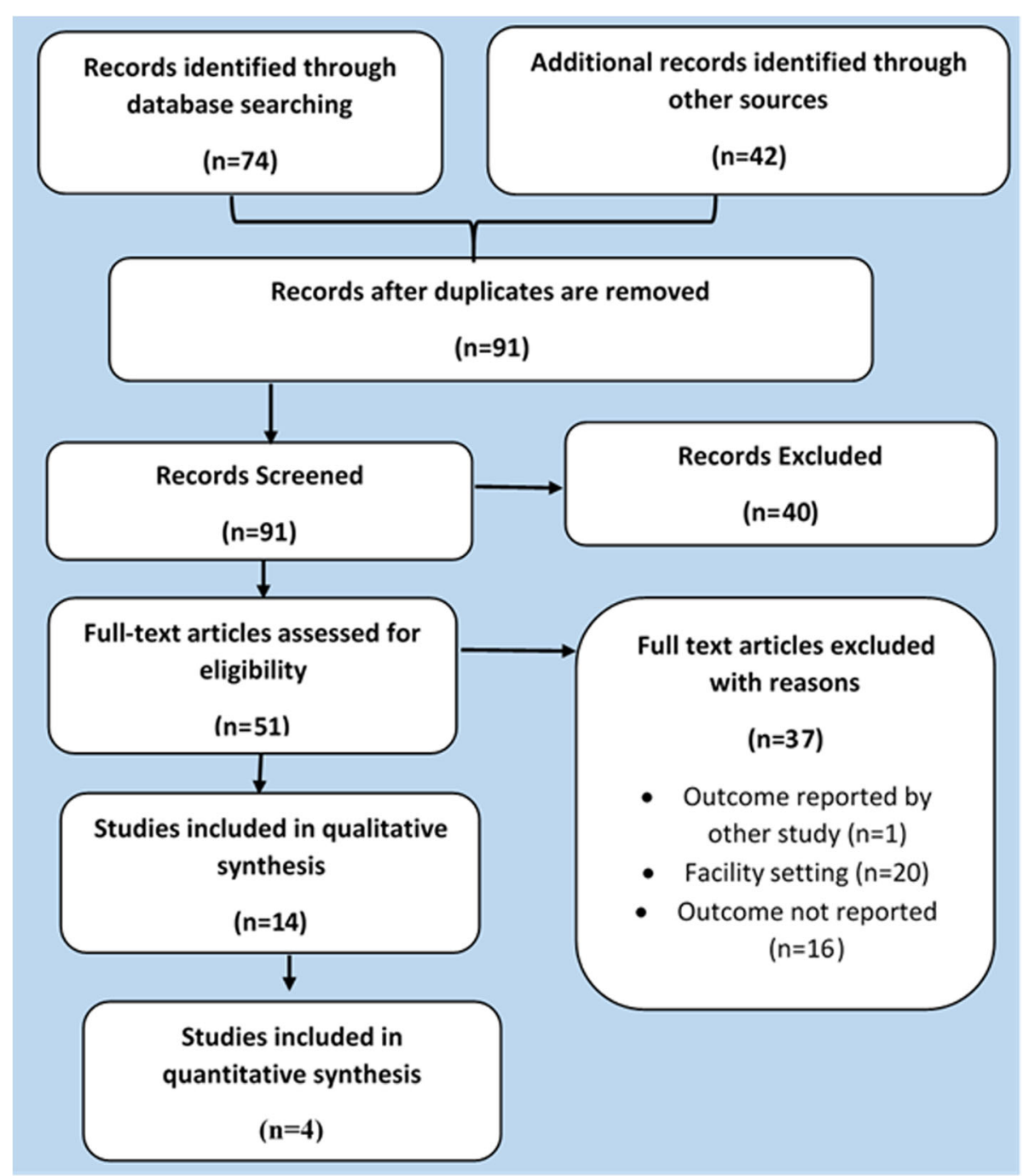

Fig. 1 Study flow diagram

hand, the cluster RCT study included scored low (46\%) where it had baseline imbalances as well as lacked masking of study of participants, personnel, and assessors [41]. All experimental and quasi-experimental studies provided adequate information about random sequence generation as well as thorough description of the interventions.

Overall, the seven cross-sectional studies scored medium quality $(70 \%)$ in which most lacked strategies to deal with confounding as well as some lacked appropriate use of statistical methods of analysis. Likewise, all qualitative studies scored medium $(65 \%)$ in which they are subjected to reporting bias in which philosophical perspectives as well as researchers' experiences, beliefs, wishes, attitudes, culture, views, and personality not stated which might bias analysis and reporting.

The results of our review are presented under three sections: 1) diversion of facility birth, 2) misuse, for purposes of either abortion or labor induction/augmentation, and 3) adverse events from misuse.

\section{Diversion of facility birth}

Eleven studies (five observational before-after studies, four experimental or quasi-experimental trials, and two qualitative study) reported on the impact on facility birth as the outcome [26, 35-42, 45, 46]. All five before-after household surveys reported increased facility delivery coverage after the intervention: four percentage points increase in Nepal [37] and Liberia [40], 11\% points in Afghanistan [36], 39\% points in Ghana [35], and 46\% points in India [45] at the end of the intervention when compared to the baseline (Fig. 2).

A quasi-experimental study in Afghanistan demonstrated an increase of 3.3 percentage points in facility birth rates comparing between the intervention and control areas $(p<0.001)$; while a RCT in India showed a 


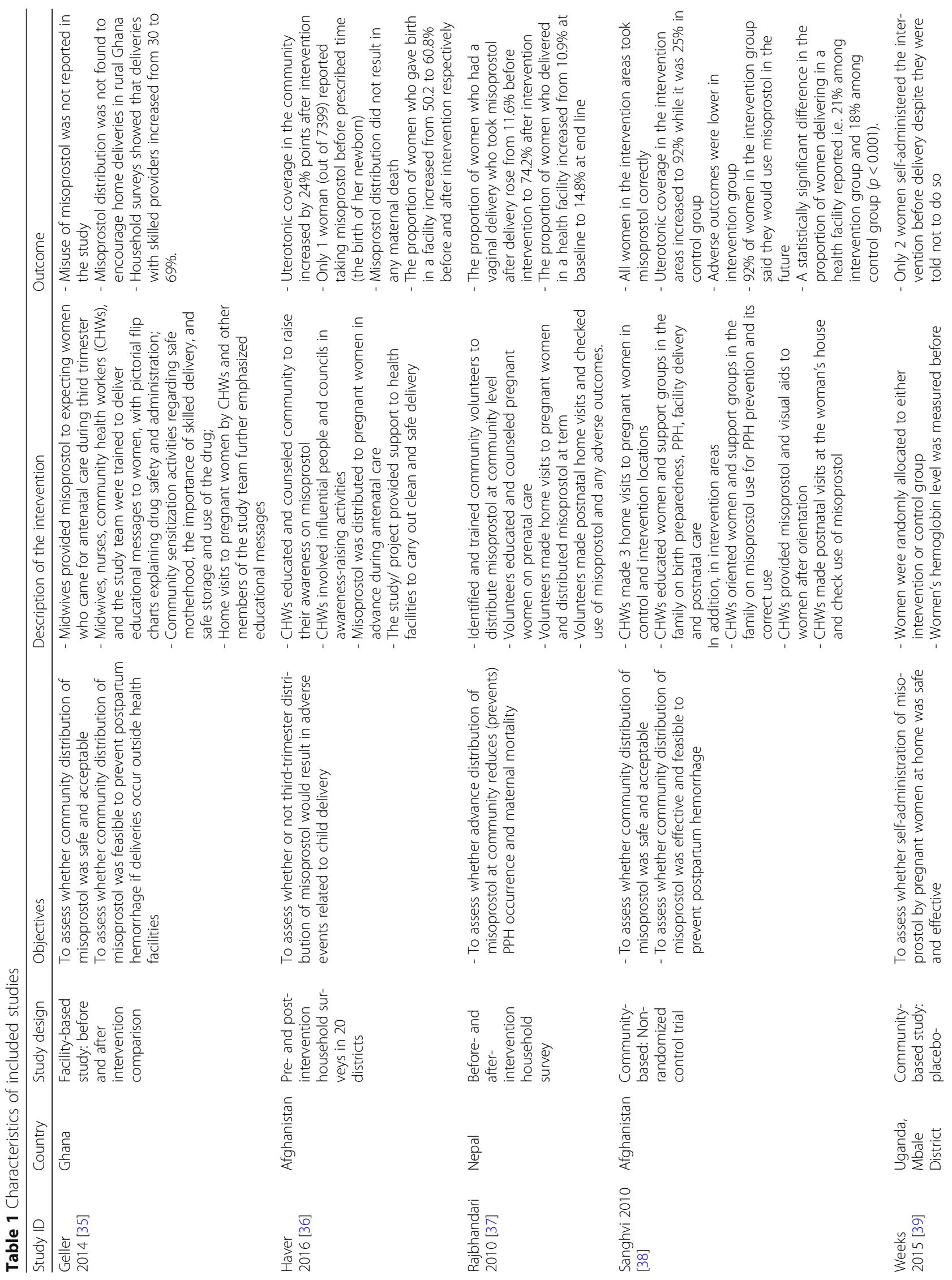




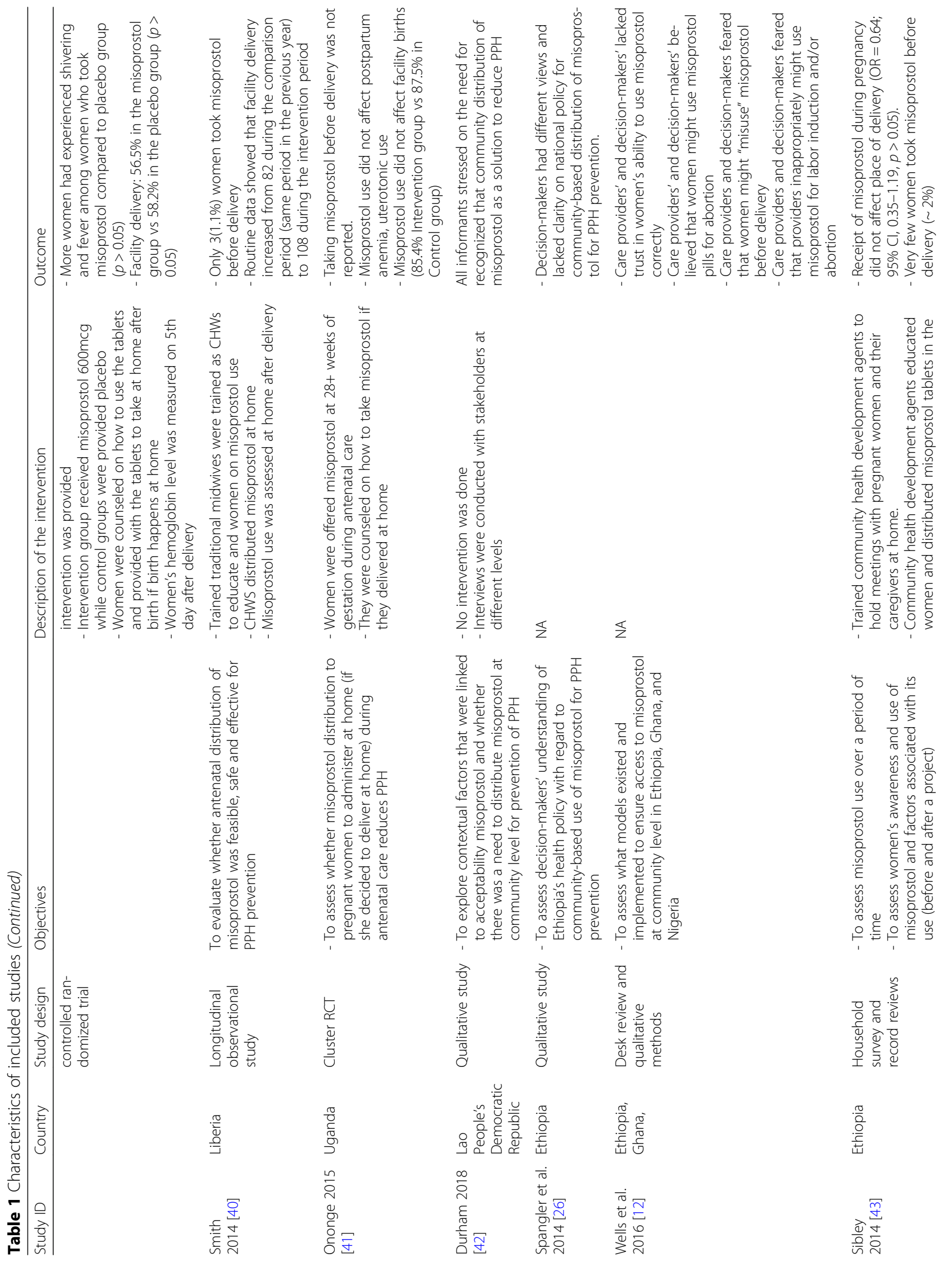


Tiruneh et al. BMC Pregnancy and Childbirth

(2019) 19:404

Page 7 of 11

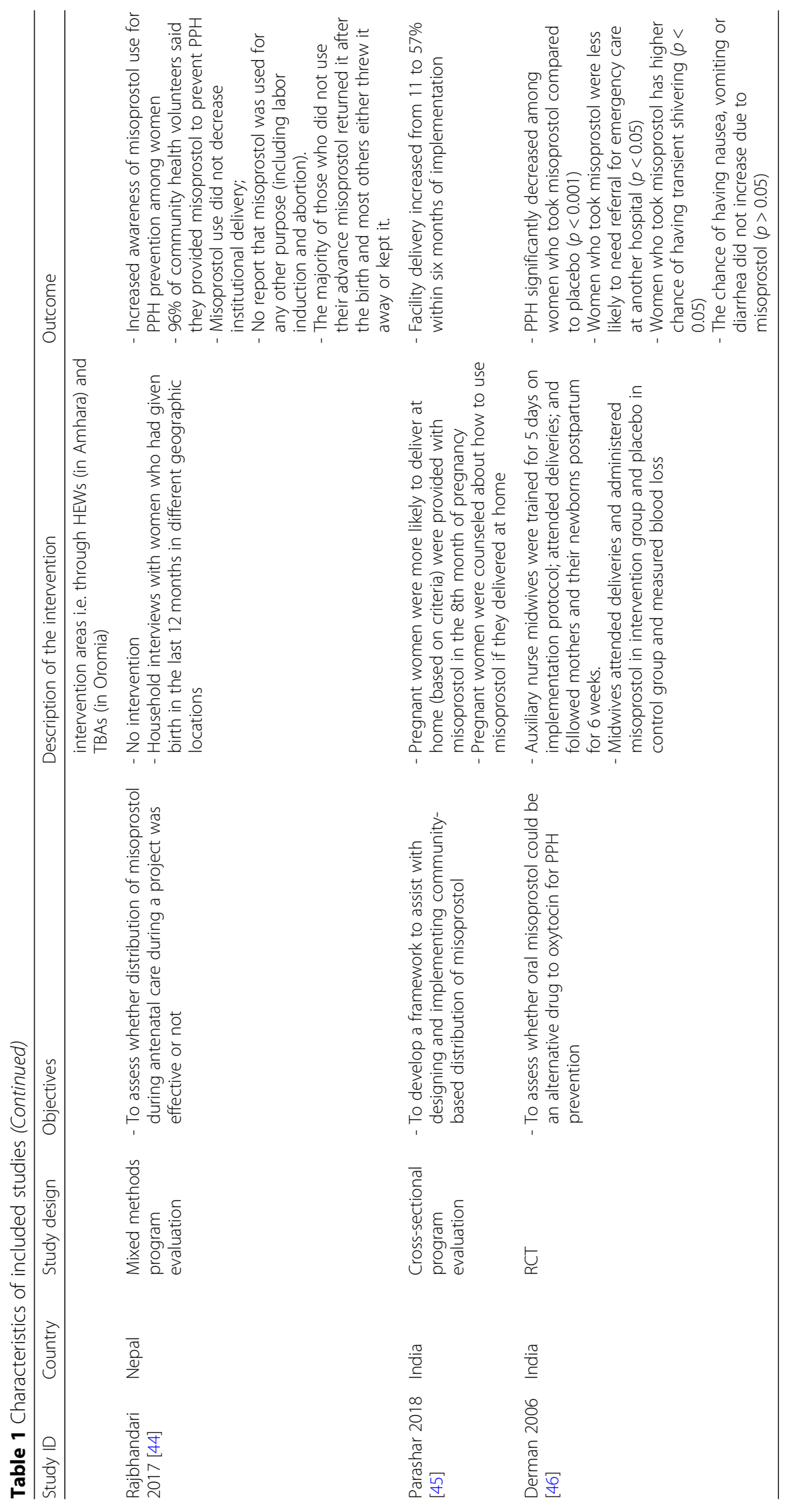




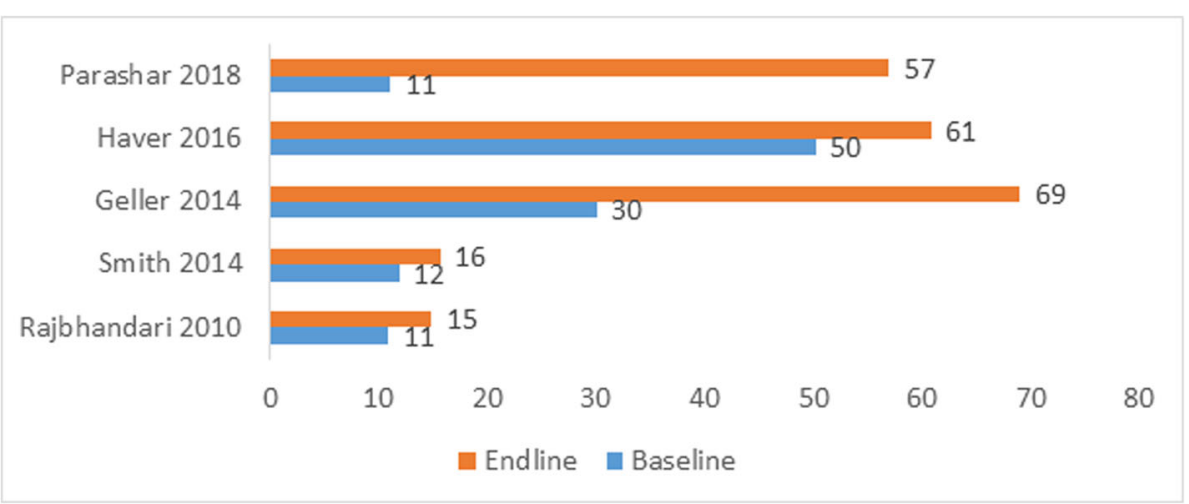

Fig. 2 Changes in facility delivery rate before and after the intervention

decrease of 1.6 percentage points $(p>0.05)$ and two cluster randomized trials in Uganda showed a decrease of 1.5 and 2.1 percentage points $(p>0.05)$ in facility birth rates, comparing between the intervention and control areas $[38,39,41]$. The pooled analysis involving 7564 women, from four of the studies, revealed that there is no significant difference in facility delivery among the advanced distribution of misoprostol and control groups [OR 1.011; 95\% CI: 0.906-1.129] (Table 2).

A qualitative study among health professionals in Laos also indicated that community distribution of misoprostol, for the prevention of $\mathrm{PPH}$, is acceptable to community members and stakeholders and it is a feasible interim solution until access to facility birth is improved. The study recognized misconceptions as barriers that might hinder community-based distribution of misoprostol [42]. Another study in Ethiopia reported regional differences in understanding the implementation strategy of misoprostol and concern among policymakers that distribution of misoprostol will be seen as encouraging home birth [26].

\section{Misuse}

A program evaluation report in Nepal showed that there was no evidence to suggest that misoprostol distributed for the purpose of the prevention of $\mathrm{PPH}$ is being misused for labor induction or pregnancy termination [44]. Moreover, as presented in Table 3, in the community-based distribution of misoprostol programs, administration of misoprostol

Table 2 Comparison of facility delivery rates between the intervention and control areas

\begin{tabular}{lllllll}
\hline Study & \multicolumn{2}{l}{ Facility delivery rate (\%) } & OR & {$[95 \% \mathrm{Cl}]$} & & $\begin{array}{l}\% \\
\text { Weight }\end{array}$ \\
\cline { 2 - 4 } & Intervention & Comparison & & & & Weight \\
\hline Sanghvi 2010 & 21.4 & 18.1 & 1.229 & 1.023 & 1.477 & 35.93 \\
Weeks 2015 & 56.5 & 58.0 & 0.940 & 0.697 & 1.269 & 13.52 \\
Ononge 2015 & 85.4 & 87.5 & 0.834 & 0.647 & 1.075 & 18.80 \\
Derman 2006 & 53.2 & 54.8 & 0.937 & 0.770 & 1.139 & 31.76 \\
I-V pooled OR & & & 1.011 & 0.906 & 1.129 & 100.0 \\
\hline
\end{tabular}

before delivery was reported in less than $2 \%(n=17)$ among seven studies involving 11,108 mothers [35, 36, 40, 41, 43].

A cluster randomized controlled trial in Uganda [41] and an operations research in Ghana [35] reported that no woman took misoprostol before their babies' birth. Another before-after study in Afghanistan reported that only 1 out of 7399 women in the study took misoprostol before the birth of her newborn [36]. Similarly, according to a trial in Uganda, only 2 out of 700 women took tablets before delivery [39]. In Liberia, only 3 of 265 women took misoprostol prior to giving birth [40]; while in Ethiopia, less than $2 \%$ of women took the tablets before birth [43] (Table 3).

Evidence also shows that most women used the misoprostol pills as instructed [35, 37, 38]; unused doses were returned after birth to the point of distribution; and most others either threw it away or kept it [35, 44]. However, qualitative studies in Ethiopia identified, lack of trust in women's capabilities to use misoprostol correctly [12] and fear of misuse $[12,26]$, as a problem limiting the expansion of the program.

\section{Adverse effects of misuse}

Three studies reported minor adverse effects following misoprostol administration [38, 39, 46]. However, no

Table 3 Percent of women who took misoprostol before delivery

\begin{tabular}{lllll}
\hline Study ID & Country & $\%$ & $\mathrm{n}$ & $\mathrm{N}$ \\
\hline Geller 2014 & Ghana & 0.00 & 0 & 102 \\
Ononge 2015 & Uganda & 0.00 & 0 & 2057 \\
Haver 2016 & Afghanistan & 0.01 & 1 & 7399 \\
Weeks 2015 & Uganda & 0.29 & 2 & 700 \\
Smith 2014 & Liberia & 1.10 & 3 & 265 \\
Sibley 2014 & Ethiopia & 1.80 & 11 & 585 \\
Total & & & 17 & 11,108 \\
\hline
\end{tabular}


adverse outcomes of misuse were reported in either of the studies reviewed.

\section{Discussions}

This review shows that community-based distribution of misoprostol programs have demonstrated increase of facility delivery coverage after the intervention in observational studies and no significant difference of facility delivery coverage in experimental and quasi-experimental studies among the misoprostol and control groups. The studies reviewed also found very few instances of administration of misoprostol before delivery, and no adverse outcomes because of misuse. While some studies have illustrated a concern held by policymakers and provider about misoprostol misuse, diversion of facility birth, and adverse effects of its misuse [12, 22, 26]; this scoping review showed that, so far, community-based distribution of misoprostol has not negatively impacted facility birth rates (in fact some studies show an increase in facility delivery) and has not resulted in misuse of the medication for uses other than $\mathrm{PPH}$ prevention. Accordingly, there is no evidence that substantiates the fear of misoprostol misuse, diversion of facility birth, and other adverse effects of its misuse. As is evident from a qualitative study in Ethiopia [26], these misconceptions arise from the health providers' perceptions rather than the actual behavior of women using community-distributed misoprostol.

In addition, evidence shows that misoprostol is safe and effective for preventing and treating PPH in remote settings where both oxytocin and timely transfer to higher-level care are not available [21, 25, 47]. Previous studies also report that community health workers or other lower-level workers are able to safely administer misoprostol [16, 42]. Women were found to have no major problem of misusing the drug and it was found to be acceptable by them [16]. Another rapid review of the literature showed that distribution of misoprostol in advance of delivery by lay health workers for self-administration was feasible and acceptable at all levelsend-user, health system, community, and policy [20,25].

Concerns by policymakers about misoprostol distribution at community level, often unsupported by available evidence [25], impedes the strategy being translated into effective policies, programs, and practice. Concerns primarily include fear of women using misoprostol for inducing abortion or labor, and diversion of facility birth to home deliveries $[25,39,48]$. In addition to policymaker resistance, there is a range of other barriers that impede access to a uterotonic for prevention of PPH for every woman. Barriers include service delivery challenges, supply and procurement, financial, national and global policy environments, and factors more closely connected to the end-user [49]. These implementation barriers represent important threats to any communitybased misoprostol distribution program, and most of these barriers are common health system weaknesses in many LMICs [20].

Community-based distribution of misoprostol is a compelling strategy to be implemented parallel to strengthening healthcare facilities to increase safe institutional deliveries $[22,25]$ and ensuring universal access to uterotonics for every woman. A review by Hobday et al. recommends simultaneously promoting facility delivery and strengthening health systems to avail misoprostol at the community level [16]. Community distribution of misoprostol is thus a complementary strategy for increasing the availability of misoprostol and actively promoting facility births through increasing contact with pregnant women. Increasing interaction with pregnant women also offers the opportunity to promote early care-seeking and referral during pregnancy [20]. As such, community-based distribution of misoprostol programs should include the promotion of facility-based birth $[4,35,42]$ as a critical intervention. Successful implementation of misoprostol distribution can be facilitated by creating an enabling environment through supportive policies, designing a formal plan for supplies, task shifting strategies, and appropriate use of guidelines and protocols [27]. Moreover, strong leadership and political commitment, training, and community mobilization were identified as critical success factors [20].

This study provides critical documentation of evidence to support policymakers and program managers to develop national policies and strategies for the implementation of community-based distribution of misoprostol to prevent $\mathrm{PPH}$ and reduce maternal mortality. It also highlights that rates of administration of misoprostol before delivery and adverse outcomes of such misuse are very low, especially when compared to the grave risks women can encounter without access to uterotonics. As such, community-based distribution of misoprostol is an appropriate strategy to be implemented while working towards achieving facility delivery as the norm.

National guidance and evidence-based policies on misoprostol distribution initiated by higher levels of the health system can facilitate reassuring reluctant policymakers and providers who hold persistent, but unfounded, fears of misuse and negative consequences. Creating opportunities for reflective discussions or policy dialogue is thus important for virtuous public health practice.

This review has some limitations. First, there may be possibility of missing some relevant studies due to the inclusion of only published studies and exclusion of studies published in a language other than English. Second, we found a small number of articles meeting the inclusion criteria and few rigorous studies directly investigated the negative effect of community availability of misoprostol on institutional delivery, misuse and adverse effects from misuse as a primary outcome. Accordingly, we could not 
be able to combine all the results in a meta-analysis and show pooled estimates.

\section{Conclusions}

Community-based distribution of misoprostol programs have been associated with an increase in coverage of facility-based births. This review found very few instances of administration of misoprostol before delivery, and no adverse outcomes of misuse in any of the studies reviewed.

Fears of misuse of misoprostol and increased adverse pregnancy outcomes if distributed at community level are not supported by evidence. Therefore, communitybased distribution of misoprostol can be an appropriate strategy for reducing maternal deaths caused by postpartum hemorrhages, especially in resource-limited settings where many deliveries take place outside of health facilities.

\section{Abbreviations}

AMTSL: Active Management of the Third Stage of Labor; CHW: Community Health Workers; JBI: Joanna Briggs Institute; MoH: Ministry of Health; PPH: Postpartum Hemorrhage; PRISMA: Preferred Reporting Items for Systematic Reviews and Meta-Analyses; RAC: Research Advisory Council; RCT : Randomized Controlled Trial; RMNCAH-N: Reproductive, Maternal, Newborn, Child, Adolescent Health, and Nutrition; WHO: World Health Organization

\section{Acknowledgments}

We conducted this study in response to the national Reproductive, Maternal, Newborn, Child, Adolescent Health, and Nutrition (RMNCAH-N) Research Advisory Council (RAC) of the Ministry of Health $(\mathrm{MoH})$ of Ethiopia's request to produce evidence to inform the maternal health program. We would like to acknowledge the $\mathrm{MoH}$ for giving us this opportunity. The authors are grateful to Harvard University/Fenot Project for sponsoring the Report Writing Workshop. Researchers would also like to acknowledge Adey Abebe, Communication Specialist at JSI Research and Training Institute Inc., and Sarah Hurlburt, Deputy Director of Implementation for the Fenot project, for editing this manuscript.

\section{Authors' contributions}

GT, BT, WM, YT, and AM conceptualized the paper. GT, MY, EG, and YT performed article search, data extraction, and data analysis. GT, BY, AM, WM, YT did interpretation and critical review. All authors contributed to the interpretation, commented on multiple versions, and approved the final manuscript.

\section{Funding}

The authors declare that they did not receive funding for this research from any source.

\section{Availability of data and materials}

The datasets used and/or analyzed during the current study are available from the corresponding author on reasonable request.

\section{Ethics approval and consent to participate}

Not applicable.

\section{Consent for publication}

Not applicable.

\section{Competing interests}

The authors declare that they have no competing interests.

\section{Author details}

${ }^{1}$ JSI Research \& Training Institute, Inc./ The Last Ten Kilometers (L10K) Project, Addis Ababa, Ethiopia. ${ }^{2}$ Members of the National Reproductive, Maternal,
Newborn, Child, Adolescent Health, and Nutrition (RMNCAH-N) Research Advisory Council (RAC), Addis Ababa, Ethiopia. ${ }^{3}$ Department of Global Health and Population /Fenot Project, Harvard T.H. Chan School of Public Health, Addis Ababa, Ethiopia. ${ }^{4}$ Addis Ababa University School of Public Health, Addis Ababa, Ethiopia. ${ }^{5}$ Jhpiego/HRH Project, Addis Ababa, Ethiopia. ${ }^{6}$ School of Public Health, College of Health Sciences, Mekelle University, Mekelle, Ethiopia. ${ }^{7}$ Federal Ministry of Health, Addis Ababa, Ethiopia.

Received: 7 August 2019 Accepted: 26 September 2019

Published online: 06 November 2019

\section{References}

1. WHO. Trends in Maternal Mortality: 1990 to 2015 Estimates by WHO, UNICEF, UNFPA. In: World Bank Group and the United Nations Population Division. Geneva: World health Organization; 2015.

2. WHO. Strategies toward ending preventable maternal mortality (EPMM). Geneva: World Health Organization; 2015.

3. Chou D, Daelmans B, Jolivet R, Kinney M, Say L. Ending preventable maternal and newborn mortality and stillbirths. Br Med J. 2015;351. https:// doi.org/10.1136/bmj.h4255.

4. Robinson N, Kapungu C, Carnahan L, Geller S. Recommendations for scaleup of community-based misoprostol distribution programs. Int J Gynecol Obstet. 2014;125(3):285-8.

5. Say L, Chou D, Gemmill A, Tunçalp Ö, Moller A-B, Daniels J, et al. Global causes of maternal death: a WHO systematic analysis. Lancet Glob Health. 2014;2(6):e323-e33. https://doi.org/10.1016/S2214-109X(14)70227-X.

6. Haeri S, Dildy GA. Maternal mortality from hemorrhage. Semin Perinatol. 2012;36(1):48-55. https://doi.org/10.1053/j.semperi.2011.09.010.

7. WHO. Trends in maternal mortality: 1990 to 2008. In: Estimates developed by WHO, UNICEF, UNFPA and The World Bank. Geneva: World Health Organization; 2010.

8. Mekonnen W, Gebremariam A. Causes of maternal death in Ethiopia between 1990 and 2016: systematic review with meta-analysis. Ethiopian J Health Dev. 2018;32(4):225-42.

9. EPHI. National MDSR annual report: 2008 EFY. Addis Ababa: Ethiopian Public Health Institute; 2017.

10. Lancet. WOMAN: reducing maternal deaths with tranexamic acid. Lancet. 2017;389(10084):2081. https://doi.org/10.1016/s0140-6736(17)31111-x.

11. Ronsmans C, Graham WJ. Maternal mortality: who, when, where, and why. Lancet. 2006;368(9542):1189-200. https://doi.org/10.1016/s01406736(06)69380-x.

12. Wells E, Coeytaux F, Azasi E, Danmusa S, Geressu T, McNally T, et al. Evaluation of different models of access to misoprostol at the community level to improve maternal health outcomes in Ethiopia, Ghana, and Nigeria. Int J Gynaecol Obstet. 2016;133(3):261-5. https://doi.org/10.1016/j.ijgo.2016. 04.002

13. Begley CM, Gyte GML, Devane D, McGuire W, Weeks A. Active versus expectant management for women in the third stage of labour. Cochrane Database Syst Rev. 2015;3. https://doi.org/10.1002/14651858.CD007412.pub4

14. WHO. WHO recommendations for the prevention and treatment of postpartum haemorrhage. Geneva: World Health Organization; 2012.

15. Gülmezoglu AM, Lumbiganon $\mathrm{P}$, Landoulsi S, Widmer M, Abdel-Aleem H, Festin $M$, et al. Active management of the third stage of labour with and without controlled cord traction: a randomised, controlled, non-inferiority trial. Lancet. 2012;379(9827):1721-7. https://doi.org/10.1016/S01406736(12)60206-2.

16. Hobday K, Hulme J, Belton S, Homer CS, Prata N. Community-based misoprostol for the prevention of post-partum haemorrhage: a narrative review of the evidence base, challenges and scale-up. Glob Public Health 2018;13(8):1081-97. https://doi.org/10.1080/17441692.2017.1303743.

17. Oladapo OT. Misoprostol for preventing and treating postpartum hemorrhage in the community: a closer look at the evidence. Int J Gynecol Obstet. 2012;119(2):105-10. https://doi.org/10.1016/j.jjgo.2012.08.004.

18. Prata N, Passano P, Bell S, Rowen T, Potts M. New hope: community-based misoprostol use to prevent postpartum haemorrhage. Health Policy Plan. 2012;28(4):339-46.

19. Wise A, Clark V. Strategies to manage major obstetric haemorrhage. Curr Opin Anesthesiol. 2008;21(3):281-7.

20. Smith HJ, Colvin CJ, Richards E, Roberson J, Sharma G, Thapa K, et al. Programmes for advance distribution of misoprostol to prevent post- 
partum haemorrhage: a rapid literature review of factors affecting implementation. Health Policy Plan. 2015;31(1):102-13.

21. Hofmeyr GJ, Gulmezoglu AM, Novikova N, Lawrie TA. Postpartum misoprostol for preventing maternal mortality and morbidity. Cochrane Database Syst Rev. 2013;(7):CD008982. https://doi.org/10.1002/14651858. CD008982.pub2.

22. Smith JM, Gubin R, Holston MM, Fullerton J, Prata N. Misoprostol for postpartum hemorrhage prevention at home birth: an integrative review of global implementation experience to date. BMC Pregnancy Childbirth. 2013; 13(1):44.

23. Chu CS, Brhlikova P, Pollock AM. Rethinking WHO guidance: review of evidence for misoprostol use in the prevention of postpartum haemorrhage. J R Soc Med. 2012;105(8):336-47.

24. Prata N, Mbaruku G, Grossman AA, Holston M, Hsieh K. Community-based availability of misoprostol: is it safe? Afr J Reprod Health. 2009:13(2):117-28.

25. Starrs A, Winikoff B. Misoprostol for postpartum hemorrhage: moving from evidence to practice. Int J Gynaecol Obstet. 2012;116(1):1-3. https://doi.org/ 10.1016/j.jiggo.2011.10.005

26. Spangler SA, Gobezayehu AG, Hailemariam TG, Sibley LM. Interpretation of national policy regarding community-based use of misoprostol for postpartum hemorrhage prevention in Ethiopia: a tale of two regions. J Midwifery Womens Health. 2014;59(Suppl 1):S83-90. https://doi.org/10.1111/ jmwh.12154.

27. Samnani AABA, Rizvi N, Ali TS, Abrejo F. Barriers or gaps in implementation of misoprostol use for post-abortion care and post-partum hemorrhage prevention in developing countries: a systematic review. Reprod Health. 2017:14(1):139.

28. Lockwood C, Munn Z, Porritt K. Qualitative research synthesis: methodological guidance for systematic reviewers utilizing metaaggregation. Int J Evid Based Healthc. 2015;13(3):179-87.

29. Tufanaru C, Munn Z, Aromataris E, Campbell J, Hopp L. Chapter 3: systematic reviews of effectiveness. In: Joanna Briggs Institute Reviewer's Manual. Adelaide: The Joanna Briggs Institute; 2017.

30. Moola S, Munn Z, Tufanaru C, Aromataris E, Sears K, Sfetcu R, et al. Chapter 7: systematic reviews of etiology and risk. In: Joanna Briggs Institute Reviewer's Manual. Adelaide: The Joanna Briggs Institute; 2017.

31. Tricco AC, Lillie E, Zarin W, O'Brien KK, Colquhoun H, Levac D, et al. PRISMA extension for scoping reviews (PRISMA-SCR): checklist and explanation. Ann Intern Med. 2018;169(7):467-73.

32. Sterne JA, Palmer TM. Meta-analysis in Stata: an updated collection from the Stata Journal. 2nd ed. College Station: StataCorp LP; 2016.

33. Higgins JP, Green S. Cochrane handbook for systematic reviews of interventions. Chichester: Wiley; 2011.

34. StataCorp. Stata: Release 15. In: Statistical Software. College Station: Stata Press; 2017.

35. Geller S, Carnahan L, Akosah E, Asare G, Agyemang R, Dickson R, et al. Community-based distribution of misoprostol to prevent postpartum haemorrhage at home births: results from operations research in rural Ghana. Int J Obstet Gynaecol. 2014:121(3):319-26.

36. Haver J, Ansari N, Zainullah P, Kim YM, Tappis H. Misoprostol for prevention of postpartum hemorrhage at home birth in Afghanistan: program expansion experience. J Midwifery Womens Health. 2016;61 (2):196-202.

37. Rajbhandari S, Hodgins S, Sanghvi H, McPherson R, Pradhan YV, Baqui AH. Expanding uterotonic protection following childbirth through communitybased distribution of misoprostol: operations research study in Nepal. Int J Gynecol Obstet. 2010;108(3):282-8.

38. Sanghvi H, Ansari N, Prata NJ, Gibson H, Ehsan AT, Smith JM. Prevention of postpartum hemorrhage at home birth in Afghanistan. Int J Gynecol Obstet. 2010;108(3):276-81. https://doi.org/10.1016/j.ijgo.2009.12.003.

39. Weeks AD, Ditai J, Ononge S, Faragher B, Frye LJ, Durocher J, et al. The MamaMiso study of self-administered misoprostol to prevent bleeding after childbirth in rural Uganda: a community-based, placebo-controlled randomised trial. BMC Pregnancy Childbirth. 2015;15:219. https://doi.org/10. 1186/s12884-015-0650-9

40. Smith JM, Baawo SD, Subah M, Sirtor-Gbassie V, Howe CJ, Ishola G, et al. Advance distribution of misoprostol for prevention of postpartum hemorrhage (PPH) at home births in two districts of Liberia. BMC Pregnancy Childbirth. 2014:14(1):189.

41. Ononge S, Campbell OM, Kaharuza F, Lewis JJ, Fielding K, Mirembe F. Effectiveness and safety of misoprostol distributed to antenatal women to prevent postpartum haemorrhage after child-births: a stepped-wedge cluster-randomized trial. BMC Pregnancy Childbirth. 2015;15:315. https://doi. org/10.1186/s12884-015-0750-6

42. Durham J, Phengsavanh A, Sychareun V, Hose I, Vongxay V, Xaysomphou D, et al. Misoprostol for the prevention of postpartum hemorrhage during home births in rural Lao PDR: establishing a pilot program for community distribution. Int J Womens Health. 2018;10:215-27. https://doi.org/10.2147/ IJWH.S150695.

43. Sibley LM, Spangler SA, Barry D, Tesfaye S, Desta BF, Gobezayehu AG. A Regional Comparison of Distribution Strategies and Women's Awareness, Receipt, and Use of Misoprostol to Prevent Postpartum Hemorrhage in Rural Amhara and Oromiya Regions of Ethiopia. J Midwifery Womens Health. 2014;59(s1):S73-82. https://doi.org/10.1111/jmwh.12136.

44. Rajbhandari SP, Aryal K, Sheldon WR, Ban B, Upreti SR, Regmi K, et al. Postpartum hemorrhage prevention in Nepal: a program assessment. BMC Pregnancy Childbirth. 2017;17(1):169. https://doi.org/10.1186/s12884-0171347-z.

45. Parashar R, Gupt A, Bajpayee D, Gupta A, Thakur R, Sangwan A, et al. Implementation of community based advance distribution of misoprostol in Himachal Pradesh (India): lessons and way forward. BMC Pregnancy Childbirth. 2018;18(1):428. https://doi.org/10.1186/s12884-018-2036-2.

46. Derman RJ, Kodkany BS, Goudar SS, Geller SE, Naik VA, Bellad MB, et al. Oral misoprostol in preventing postpartum haemorrhage in resource-poor communities: a randomised controlled trial. Lancet. 2006;368(9543):1248-53. https://doi.org/10.1016/s0140-6736(06)69522-6.

47. Oladapo OT, Fawole B, Blum J, Abalos E. Advance misoprostol distribution for preventing and treating postpartum haemorrhage. Cochrane Database Syst Rev. 2012;(2). https://doi.org/10.1002/14651858.CD009336.pub2.

48. Collins L, Mmari K, Mullany LC, Gruber CW, Favero R. An exploration of village-level uterotonic practices in Fenerive-Est, Madagascar. BMC Pregnancy Childbirth. 2016;16(1):69. https://doi.org/10.1186/s12884-0160858-3.

49. Smith HJ, Colvin CJ, Richards E, Roberson J, Sharma G, Thapa K, et al. Programmes for advance distribution of misoprostol to prevent postpartum haemorrhage: a rapid literature review of factors affecting implementation. Health Policy Plan. 2016;31(1):102-13. https://doi.org/10. 1093/heapol/czv012.

\section{Publisher's Note}

Springer Nature remains neutral with regard to jurisdictional claims in published maps and institutional affiliations.
Ready to submit your research? Choose BMC and benefit from:

- fast, convenient online submission

- thorough peer review by experienced researchers in your field

- rapid publication on acceptance

- support for research data, including large and complex data types

- gold Open Access which fosters wider collaboration and increased citations

- maximum visibility for your research: over $100 \mathrm{M}$ website views per year

At $\mathrm{BMC}$, research is always in progress.

Learn more biomedcentral.com/submissions 